\title{
$\$$ Research Square \\ Morbidity and mortality in the antiretroviral era in sub-Saharan Africa: a systematic review protocol
}

Manimani Riziki Ghislain ( $\triangle$ manimaniriziki1@gmail.com )

University of KwaZulu-Natal College of Health Sciences https://orcid.org/0000-0003-4053-0236

AGANZE Gloire-Aime MUSHEBENGE

University of KwaZulu-Natal College of Health Sciences

NOMBULELO MAGULA

University of KwaZulu-Natal College of Health Sciences

\section{Protocol}

Keywords: Morbidity, Mortality, Antiretroviral treatment, Sub-Sahara Africa

Posted Date: February 17th, 2020

DOI: https://doi.org/10.21203/rs.2.23685/v1

License: (1) This work is licensed under a Creative Commons Attribution 4.0 International License.

Read Full License 


\section{Abstract}

\section{Background}

Worldwide, Human Immunodeficiency Virus / Acquired Immunodeficiency Syndrome (HIV/AIDS) remains a public health problem. The prevalence of HIV in Sub-Saharan Africa is one of the uppermost in the world.

Method/design

Observational studies will be systematically reviewed reporting on morbidity and mortality in the antiretroviral therapy (ART) era in Sub-Saharan Africa. We will search relevant studies from the following databases: PubMed, Medline, CINAHL. Two review authors will independently screen titles abstracts and full text articles in duplicate, extract data and assess the bias. Discrepancies will be resolved by discussion or arbitration of a third review author. The study will use the Preferred Reporting Item of Systematic Review (PRISMA 2015) guideline.

Discussion

This review will summarise the determinants of morbidity and causes of mortality in the antiretroviral era in Sub-Saharan Africa. The findings of this study will help to improve opportunistic infection's prevention and clinical outcomes in ART era.

Systematic review registration

PROSPERO CDR42019141933

\section{Background}

Worldwide Human Immunodeficiency Virus (HIV) / Acquired Immunodeficiency Syndrome (AIDS) is one of the major causes of death with an estimation of 3.1 million of death each year [1]. Approximately 36.9 million persons in the world are living with HIV, and 1.8 million persons were newly infected in 2017 [2]. Moreover, it has been reported that 940,000 persons worldwide died from AIDS-related illnesses in 2017 [2].

Sub-Saharan Africa is the region hardest hit by HIV and AIDS in the World. The Southern African Development Community (SADC) countries are generally most affected by HIV and AIDS [3]. According to some authors, there are different factors that make African population more susceptible to HIV and AIDS in the world. Among them, concurrent or simultaneous sexual intercourse practices by African men is argued as a major role player in the vast spread of HIV in Sub-Saharan African countries as opposed to high-income countries where there are serial monogamy practices [4]. 
Other authors argued that political instability, underdevelopment, widespread poverty and poor infrastructure are the major reasons for the rapid spread of HIV in African countries [5].

Sub-Saharan Africa has the biggest share of the 40 million people currently living with HIV and AIDS in the world [6]. In 2014 studies reported that 25.8 (24.0-28.7) million people were estimated to be living with HIV, the region accounted for nearly $70 \%$ of new infections worldwide [6]. According to UNAIDS (2018), 300.000 men and 270.000 women died of AIDS-related illnesses in Sub-Saharan Africa in 2017 [7].

Antiretroviral therapy (ART) has decreased morbidity and mortality among people living with HIV [8]. However, despite the availability of antiretroviral therapy (ART), a substantial portion of HIV infected patients have continued to be hospitalised and die from both AIDS-related and non- AIDS-related causes [9].

Therefore, this systematic review seeks to identify the literature available, the research gaps, on the morbidity and mortality in the antiretroviral treatment (ART) Era in Sub Saharan Africa and suggest novel ideas for future research.

\section{Research Question}

\section{Main research question}

What are the determinants of morbidity and specific causes of mortality in the antiretroviral treatment (ART) Era in Sub-Saharan Africa?

\section{Specific research question}

1. What are the research gaps on morbidity and mortality in the antiretroviral treatment (ART) Era in Sub-Saharan Africa?

2. What are the reasons for hospitalizations in the antiretroviral treatment (ART) Era in Sub-Saharan Africa?

3. What are the causes of death in the antiretroviral treatment (ART) Era in Sub-Saharan Africa?

\section{Aims And Objectives Of The Study \\ Main aim}

To identify the determinants of morbidity and specific causes of mortality in the antiretroviral treatment (ART) Era in Sub-Saharan Africa.

\section{Objectives}


- Search the literature to identify available literature or the research gaps on the morbidity and mortality in the antiretroviral treatment (ART) Era in Sub-Saharan Africa.

- Search the literature to identify the reasons for hospitalizations in the antiretroviral treatment (ART) Era in Sub-Saharan Africa.

- Search the literature to determine the causes of mortality in the antiretroviral treatment (ART) Era in Sub-Saharan Africa.

\section{Methods}

We are going to follow the Preferred Reporting Items for the Systematic Reviews and Meta-analysis Protocols (PRISMA-P) 2015 guideline (additional file1) [10]. We will systematically review observational studies reporting on morbidity and mortality in the ART era in Sub-Saharan Africa.

\section{Participant}

Participants included in eligible studies will be adult patients, 18 years or older infected with HIV during the antiretroviral therapy.

\section{Interventions}

The antiretroviral therapy (ART) used to treat people with HIV infection in Sub-Saharan Africa from 2008 to 2018.

\section{Comparison}

Not planned

\section{Outcomes}

The reasons for hospitalization and the causes of mortality among people with HIV infection, dying in the antiretroviral era will be described and that will help clinicians and patients living with HIV to have comprehensive information and find strategies to improve treatment outcomes.

\section{Eligibility criteria}

Inclusion criteria

- Studies reporting on morbidity and mortality in the antiretroviral era in Sub-Saharan Africa.

- Studies conducted in the period of 2008 to 2018. 
- Studies reporting on Adult males or females aged 18 years or older.

- Peer-reviewed English language publications

- Observational studies on Sub-Saharan Africa.

\section{Exclusion Criteria}

- Studies reporting on morbidity or mortality in Pregnant women infected with HIV

- Studies reporting on morbidity or mortality in women infected with HIV in the post-partum period.

\section{Search Strategy for identifying relevant studies}

To identify relevant studies, we will search in the following database: Medline, Pub Med, CINAHL. Studies published in English from January 2008 to December 2018 in Sub-Saharan Africa. The choice from 2008 to 2018 because we want to compare the time the ART was available for everyone and the time it was not available for everyone.

The search strategy will be based on a combination of relevant terms.

Find below the main search strategy conducted in PubMed in Table1.

Table 1: Search strategy in PubMed

\begin{tabular}{|ll|}
\hline Search & Search terms \\
\hline$\# 1$ & (Morbidity OR Opportunistic infection related HIV) [MeSH Terms] \\
\hline \#2 & (Mortality OR Death) [MeSH Terms] \\
$\# 3$ & (ART OR Antiretroviral therapy) [MeSH Terms] \\
$\# 4$ & (Sub-Saharan Africa) [Title/Abstract] \\
\hline 1 AND \#2 AND \#3 AND \#4 \\
\hline
\end{tabular}

We will adapt this search strategy for a possible extension to other databases and it will be adapted as we progress through the review. We will also contact experts in the field to identify additional eligible studies and we will manually search reference lists from relevant studies.

\section{Data collection and analysis}

Two reviewers will follow the inclusion criteria for selecting studies, articles will be identified and screened by their titles and abstracts eligibility. The full texts of articles will be retrieved. The process of 
literature selection and reasons for exclusion and inclusion will be documented by a PRISMA (Preferred Reporting Items for Systematic Review and Meta-Analysis) flow diagram (figure 1) [11].

\section{Data extraction and management}

Data will be extracted in accordance with the methods outlined in the Cochrane Handbook for systematic reviews of interventions and the data extraction form will be designed and it will be collected in the following order:

- First author-name

- Years of publication,

- Geographical location

- Sample size

- Participants (age, sex)

- Study design

- Intervention

- Outcome

- Conclusion

If there are disagreements between the two review authors, it will be resolved through discussion and by consulting a third author. For managing missing data, we will contact the corresponding author of the respective studies to obtain the required details.

\section{Data analysis and synthesis.}

We will systematically review observational studies that examined morbidity and mortality in antiretroviral therapy in Sub-Saharan Africa. All statistical analyses will be carried out using Stata statistical software version 14 (Stata Corporation, College Station, Texas, USA). Statistical heterogeneity among the included studies will be assessed by the $X^{2}$ test on Cochrane's $Q$ statistic. Significant heterogeneity will be indicated if $P$-value is less than 0.1 . Heterogeneity will be quantified by using the Isquared values. Values of 25,50 , and $75 \%$ for I-squared will be represented low, medium, and high heterogeneity, respectively [12]. If substantial heterogeneity is detected, a subgroup will be performed to investigate the possible sources of heterogeneity using the following grouping variables: gender (male, female).

A qualitative narrative synthesis of the findings will be performed, $R$ software will be used to synthesis the data, we will capture information into a spreadsheet about the most causes of hospitalization and death related to HIV in the antiretroviral treatment, Graphic displays such as Bar chart or multiple bar chart 
component will be used to visually compare the prevalence of comorbidities across the study region. This will also enable to provide any form of patterns in the comorbidities.

\section{Subgroup analysis}

A subgroup analysis will be conducted following Gender (male vs female) The heterogeneity between subgroups will be detected by using the $X^{2}$ test on Cochrane's $Q$ statistic.

\section{Risk Of Bias Assessment And Quality Of Evidence}

Two reviewers (MRG and GAM) will independently perform each quality assessment. Differences in ratings will be resolved through discussion. We are going to use Cochrane risk of bias tool to assess publication bias, addressing assessments for random sequence generation, concealment of allocation, blinding and the outcome measurements, and completeness of outcome reporting [13]. The value of the low, high or unclear risk of bias will be used for the included study. To assess the statistical significance of publication bias across studies we will use Visual assessment of the funnel plot and the Egger's statistic [14].

The Mixed Methods Appraisal Tool (MMAT) version 2018 will be used to assess the quality of evidence for each outcome [15]. This tool will allow us to assess the appropriateness and the quality of research. Studies will be scored following the specific criterion. The tool permits to appraise the methodological quality of five categories to studies: qualitative research, randomized controlled trials, non-randomized studies, quantitative descriptive studies, and mixed methods studies.

\section{Discussion}

Antiretroviral treatment (ART) is known for improving the quality of life for infected individuals, reducing opportunistic infections and AIDS-related mortality [16]. It was anticipated initially that the dramatic scale-up of ART would result in clinics and services becoming over-stretched and have a negative quality of care, however after one-year studies have shown that there was no significant effect on patients results related to the increase in antiretroviral therapy provision, either in terms of either morbidity or mortality [17]. This study will systematically review articles reported in Sub-Saharan Africa on the morbidity and mortality in the antiretroviral therapy from 2008 to 2018, following the inclusion and exclusion criteria.

\section{Abbreviations}

ART: Antiretroviral therapy; HIV/AIDS: Human Immunodeficiency Virus / Acquired Immunodeficiency Syndrome PICO: Population, Intervention, Comparator and Outcome; PRISMA: Preferred Reporting Items for Systematic Reviews and Meta-Analyses; SADC: Southern African Development Community; UKZN: 
University of KwaZulu-Natal; GRADE: Grading of Recommendations Assessment, Development, and Evaluation

\section{Declarations}

\section{Acknowledgements}

The authors would like to acknowledge and thank the Nelson R Mandela School of the College of Health Sciences, University of KwaZulu-Natal, for the support. I am grateful to the statistician: Partson Tinarwo and librarians of medical school for their assistance.

\section{Funding}

This review is not being funded.

\section{Availability of data and materials}

All data generated from this study will be included in the published systematic review article and will also be available on request.

\section{Authors' contributions}

MRG conceptualised the study and prepared the manuscript under the guidance and supervision of NM. AGAM revised the draft for its intellectual content. All authors contributed to the development and design of the study. MRG and BN contributed to the methodology and reviewing of the manuscript. All authors contributed to the final version. All authors read and approved the final manuscript.

\section{Ethics approval and consent to participate}

Not applicable.

\section{Consent for publication}

Not applicable.

\section{Competing interests}

The authors declare no competing interests. 


\section{Author's information}

${ }^{1} \mathrm{MRG}$ is a master's student in the Discipline of Internal Medicine, College of Health Sciences, Medical School Campus, University of KwaZulu-Natal, P B X54001, Durban 4000, South Africa

\section{References}

1. Etard J-F, Ndiaye I, Thierry-Mieg M, Guèye NFN, Guèye PM, Lanièce I, Dieng AB, Diouf A, Laurent C, Mboup S: Mortality and causes of death in adults receiving highly active antiretroviral therapy in Senegal: a 7-year cohort study. J Aids 2006, 20(8):1181-1189.

2. Mitruka K, Bamrotiya M, Agarwal R, Parvez A, Allam RR, Sivalenka S, Deoraj P, Prasad R, Devi U, Keskar P: Implementation of the Treat All Policy Among Persons with HIV Infection Enrolled in Care But Not on Antiretroviral Therapy-India, May 2017-June 2018. J Morbidity Mortality Weekly Report 2018, 67(47):1305.

3. Jooma MB: Southern Africa assessment: food security and HIV/AIDS. J African Security Studies 2005, 14(1):59-66.

4. Crane J: Explaining the Epidemic-A review of Helen Epstein, The invisible cure: Africa, the West, and the fight against AIDS. New York: Farrar Straus Giroux, 2007. J BioSocieties 2009, 4(1):103-107.

5. George E: The human right to health and hiv/aids: South africa and south-south cooperation to reframe global intellectual property principles and promote access to essential medicines. $J$ Indiana Journal of Global Legal Studies 2011, 18(1):167-197.

6. Ethiopia F: Country Progress Report on the HIV Response. 2014.

https://www.unaids.org/sites/default/files/country/documents/ETH_narrative_report_2014.pdf. Accessed on 12 February 2020

7. Sidibé M: UNAIDS Data 2018. J UNAIDS 2018. http://www. unaids.

org/sites/default/files/media_asset/unaids-data-_en. pdf. Accessed 12 February 2020

8. Mocroft A, Ledergerber B, Katlama C, Kirk O, Reiss P, d, Monforte AdA, Knysz B, Dietrich M, Phillips A, Lundgren JD: Decline in the AIDS and death rates in the EuroSIDA study: an observational study. $J$ The Lancet 2003, 362(9377):22-29.

9. Smith CJ, Sabin CA, Lundgren JD, Thiebaut R, Weber R, Law M, D'ARMINIO MONFORTE A, Kirk O, Friis-Moller N, Phillips A: Factors associated with specific causes of death amongst HIV-positive individuals in the D: A: D study: Erratum. J Aids 2011, 25(6).

10. Moher D, Shamseer L, Clarke M, Ghersi D, Liberati A, Petticrew M, Shekelle P, Stewart LA: Preferred reporting items for systematic review and meta-analysis protocols (PRISMA-P) 2015 statement. $J$ Systematic reviews 2015, 4(1):1.

11. Stewart LA, Clarke M, Rovers M, Riley RD, Simmonds M, Stewart G, Tierney JF: Preferred reporting items for a systematic review and meta-analysis of individual participant data: the PRISMA-IPD statement. J Jama 2015, 313(16):1657-1665. 
12. Higgins JP, Thompson SG: Quantifying heterogeneity in a meta-analysis. J Statistics in medicine 2002, 21(11):1539-1558.

13. Higgins J, Altman D, Gøtzsche P, Jüni P, Moher D, Oxman A, Savovic J, Schulz K, Weeks L, Sterne J: Cochrane bias methods group; cochrane statistical methods group. $J$ The Cochrane Collaboration's tool for assessing risk of bias in randomised trials BMJ 2011, 343(7829):d5928.

14. Egger M, Smith GD, Schneider M, Minder C: Bias in meta-analysis detected by a simple, graphical test. J Bmj 1997, 315(7109):629-634.

15. Hong QN, Fàbregues S, Bartlett G, Boardman F, Cargo M, Dagenais P, Gagnon M-P, Griffiths F, Nicolau B, O'Cathain A: The Mixed Methods Appraisal Tool (MMAT) version 2018 for information professionals and researchers. J Education for Information 2018, 34(4):285-291.

16. Louwagie GM, Bachmann MO, Meyer K, le R Booysen F, Fairall LR, Heunis C: Highly active antiretroviral treatment and health related quality of life in South African adults with human immunodeficiency virus infection: A cross-sectional analytical study. J BMC public health 2007, 7(1):244.

17. Cornell M, Johnson LF, Wood R, Tanser F, Fox MP, Prozesky H, Schomaker M, Egger M, Davies MA, Boulle A: Twelve-year mortality in adults initiating antiretroviral therapy in South Africa. J Journal of the International AIDS Society 2017, 20(1):21902.

\section{Figures}




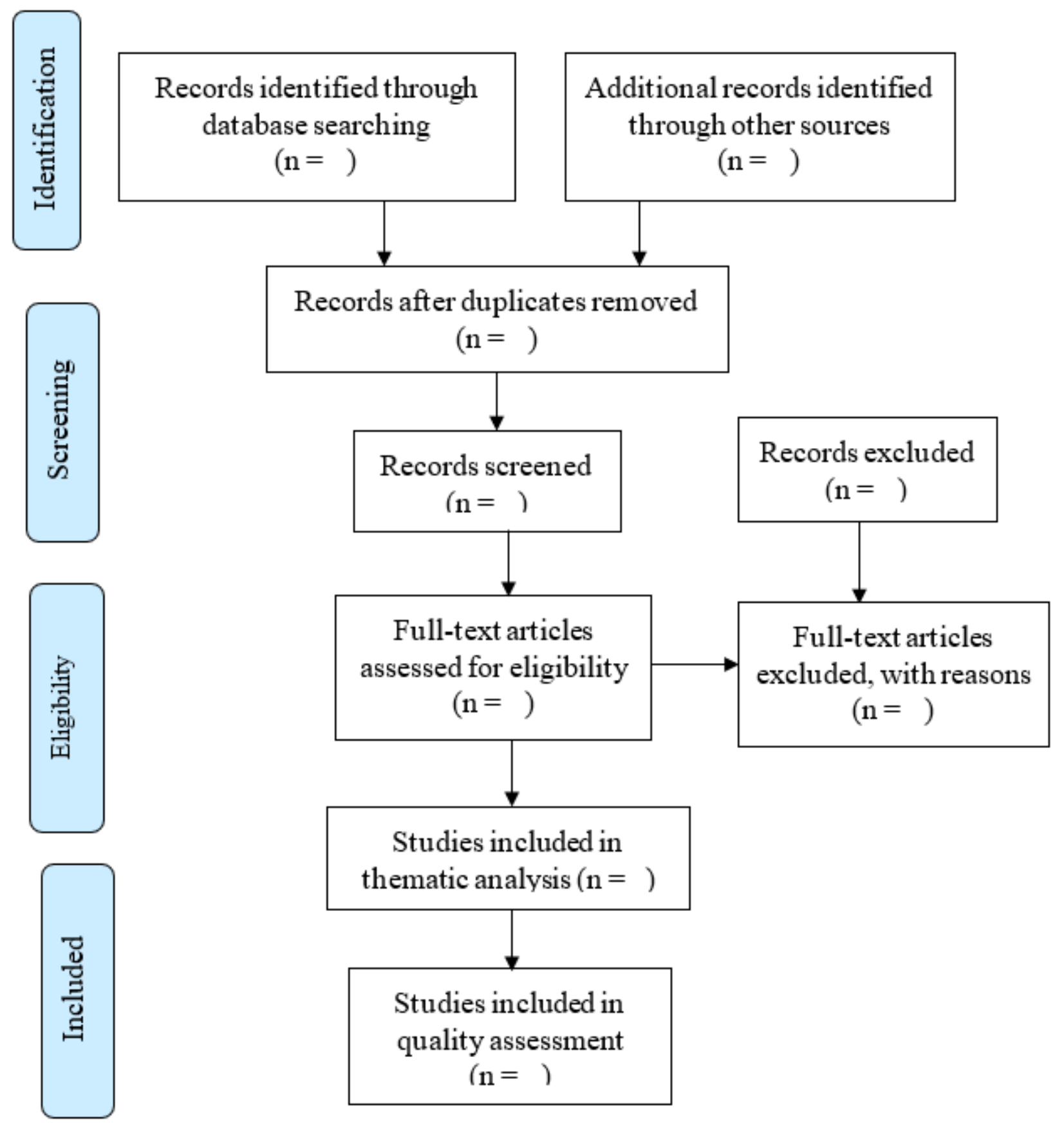

Figure 1

The PRISMA Flow Diagram for the systematic review screening process

\section{Supplementary Files}

This is a list of supplementary files associated with this preprint. Click to download.

- ManimaniPRISMAPCHECKLISTPROTOCOL.docx 